\title{
THE IMPORTANCE OF JET BENDING IN GAMMA-RAY AGNs-REVISITED
}

\author{
P. J. GRaham ${ }^{1,2}$ and S. J. TingaY ${ }^{2}$ \\ ${ }^{1}$ School of Physics, University of New South Wales, Sydney, NSW 2052, Australia \\ 2 International Centre for Radio Astronomy Research, Curtin University, Bentley, WA, Australia \\ Received 2013 May 22; accepted 2014 January 22; published 2014 March 18
}

\begin{abstract}
We investigate the hypothesis that $\gamma$-ray-quiet active galactic nuclei (AGNs) have a greater tendency for jet bending than $\gamma$-ray-loud AGNs, revisiting the analysis of Tingay et al. We perform a statistical analysis using a large sample of 351 radio-loud AGNs along with $\gamma$-ray identifications from the Fermi Large Area Telescope (LAT). Our results show no statistically significant differences in jet-bending properties between $\gamma$-ray-loud and $\gamma$-ray-quiet populations, indicating that jet bending is not a significant factor for $\gamma$-ray detection in AGNs.
\end{abstract}

Key words: galaxies: active - galaxies: jets - gamma rays: general - quasars: general

Online-only material: machine-readable tables

\section{INTRODUCTION}

The Energetic Gamma-Ray Experiment Telescope (EGRET) detected 66 active galactic nuclei (AGNs) with $\gamma$-ray emission at energies greater than $100 \mathrm{MeV}$ (Mattox et al. 1997a; Hartman et al. 1999). Almost all of the AGNs identified were blazars, triggering immense interest and prompting a series of studies focused on the multi-wavelength properties of these AGNs. EGRET's successor, the Large Area Telescope (LAT; on board the Fermi Gamma-Ray Space Telescope), has identified over $1000 \gamma$-ray sources with energies greater than $100 \mathrm{MeV}$ (Ackermann et al. 2011b), revealing the $\gamma$-ray sky in detail. The LAT instrument presents the opportunity to revisit questions first examined in the EGRET era with far superior data.

EGRET's discovery opened a new direction in investigating the nature and physical processes of blazars. Blazars are radio sources characterized by a compact core and relativistic jets that are aligned at a small angle to our observational line of sight, i.e., flat-spectrum radio quasars and BL Lac objects (Fichtel et al. 1994; Impey 1996; Mattox et al. 1997b). It is supposed that $\gamma$-ray emission from AGNs is produced from inverseCompton (IC) processes occurring within the jets and becomes apparently enhanced via relativistic beaming (Mattox et al. 1997b). Although there is good support for this scenario, several questions remain, particularly with respect to the origin of $\gamma$-ray production within the jets (Jorstad et al. 2001; Lähteenmäki \& Valtaoja 2003; Sikora et al. 2009), as well as how IC processes act to produce $\gamma$-ray emission (Sikora et al. 2009).

Relativistic beaming (or Doppler boosting) is evidenced by other associated properties that characterize blazars: high $\gamma$-ray luminosity (which comprises a large portion of the total radiative output) (Mattox et al. 1997b); apparent superluminal motion (Jorstad et al. 2001); and rapid variability from radio to $\gamma$-ray wavelengths (Aller et al. 1996; Lähteenmäki \& Valtaoja 2003; Kovalev et al. 2009). From the non-thermal nature of this emission, it is understood that it is produced from within the AGN jets and is relativistically beamed along the line of sight. This could explain why $\gamma$-ray-loud AGNs are also radio-loud but not all radio-loud AGNs are $\gamma$-ray-loud. If $\gamma$-ray emitting regions move at faster speeds than radio emitting regions, $\gamma$-ray emission would be Doppler boosted within a narrower cone (Salamon \& Stecker 1994). Outside the $\gamma$-ray beaming cone and within the radio beaming cone, $\gamma$-ray radiation would be Doppler dimmed, giving the appearance of a $\gamma$-ray-quiet yet radio-loud AGNs.

A second possibility suggests that the appearance of $\gamma$-ray emission may be related to AGN jet bending (von Montigny et al. 1995). If a bend in the jet occurs downstream of the $\gamma$-emitting region and upstream of regions of extended radio emission, the emissions would be Doppler-boosted in alignment to their respective sections of jet. As such, it may be possible that $\gamma$-ray emission is beamed away from us whilst radio emission is beamed toward us due to a better alignment of the radio-emitting jet section with our line of sight.

Several studies have compared $\gamma$-ray-loud and $\gamma$-ray-quiet AGN populations with respect to characteristics of the AGN core and jet components. Statistically significant differences between these populations are found for core brightness temperature, jet opening angles, and core polarization (Mattox et al. 1997b; Tingay et al. 2002; Taylor et al. 2007; Pushkarev et al. 2009; Linford et al. 2011). The distinction between populations is also established for radio flux density variability between $\gamma$-rayloud and $\gamma$-ray-quiet AGNs (Aller et al. 1996; Lähteenmäki \& Valtaoja 1999; Tingay et al. 2003; Kovalev et al. 2009; Richards et al. 2011), suggesting that radio and $\gamma$-ray emission are produced within the same region within AGN jets (Lähteenmäki \& Valtaoja 1999). Hughes et al. (2011) propose that radio-to$\gamma$-ray variability is caused by oblique shocks in the AGN jets. The role of oblique shocks has also been explored in jets on parsec (Tingay et al. 1996b) and kiloparsec scales (Balsara \& Norman 1992; Lebedev et al. 2004). Ackermann et al. (2011a) finds a correlation between $\gamma$-ray and radio flux in AGNs after consideration of biases unaccounted for in previous studies (Mücke et al. 1997). Studies have also correlated superluminal ejections and $\gamma$-ray flares in AGN jets, suggesting that $\gamma$-ray emission is produced within the parsec-scale region of the jets (Jorstad et al. 2001; Lähteenmäki \& Valtaoja 2003; Kovalev et al. 2009; Pushkarev et al. 2010). Furthermore, studies have related $\gamma$-ray emission with jet kinematics (Kellermann et al. 2004; Lister et al. 2009b; Savolainen et al. 2010), and there is evidence to suggest that FSRQs and BL Lac objects may have intrinsically different mechanisms for $\gamma$-ray emission (Ackermann et al. 2011b; León-Tavares et al. 2011; Lister et al. 2011; Nieppola et al. 2011; Linford et al. 2012).

In examining the jet-bend scenario of von Montigny et al. (1995), Tingay et al. (1998, hereafter Paper I) used EGRET 
identifications to compare $\gamma$-ray-loud and $\gamma$-ray-quiet AGNs with respect to their jet-bending characteristics. Their results showed a tendency in $\gamma$-ray-quiet sources to both have more jet bends and more pronounced jet-bend angles at parsec scales. Bower et al. (1997) did not find evidence to support this scenario, but their study was limited in only examining parsecto-kiloparsec jet misalignment angles. The jet-bend proposition was revisited in Taylor et al. (2007) and Linford et al. (2011) with inconclusive results (limited to 4 EGRET candidates and 30 LAT sources, respectively). Similarly, Linford et al. (2012) found that no significant difference in jet-bend angles between $\gamma$-ray-loud and $\gamma$-ray-quiet populations (limited to 19 LAT sources).

Utilizing LAT's superior $\gamma$-ray detection capabilities and a larger sample size, we re-examine the suggestion that jet bending may be a significant factor for the detection of $\gamma$-ray emission in AGNs. We follow the analysis of Paper I, testing the statistical significance of jet-bend angles, as well as the number of bends between $\gamma$-ray-loud and $\gamma$-ray-quiet AGNs. We use the "clean sample" of the second LAT AGN catalog (2LAC; Ackermann et al. 2011b) and very long baseline interferometry (VLBI) images accessible in the published literature or from the Radio Fundamental Catalog (RFC, version "rfc_2013d"; Beasley et al. (2002), Fomalont et al. (2003), Immer et al. (2011), Kovalev et al. (2007), Petrov et al. (2005, 2006, 2008, 2011a, 2011b), Petrov \& Taylor (2011), Petrov (2011, 2012, 2013)). We examine the statistics of two samples, a large but inhomogeneous sample and a smaller but homogeneous subsample based on Lister et al. (2009a, hereafter MOJAVE).

\section{SAMPLE DEFINITIONS}

In order to investigate the significance of jet bending between $\gamma$-ray-loud and $\gamma$-ray-quiet AGN populations, we surveyed the literature for VLBI images and measured source properties.

\subsection{The RFC-based Sample}

The most recent version of the RFC contains 8310 compact radio sources studied in 5719 VLBI observing sessions, making it the most complete catalog of radio sources with available VLBI data. We have compared the RFC with the CRATES catalog (Healey et al. 2007), a nearly uniform survey of flatspectrum radio sources $(\alpha>-0.5)$ containing flux densities and spectral indices for over 11,000 objects. By cross-referencing data from the CRATES catalog to sources in the RFC, we obtain a large pool of sources to apply selection criteria and draw upon in order to define a "RFC-based sample" for the purpose of our investigation.

There are a number of considerations in formulating our selection criteria for the RFC-based sample. A true crossidentification between radio/X-ray and $\gamma$-ray counterparts can only be determined when there is a correlation in variability in both bands. In practice, cross-identifications were made only for 28 sources in the 2 yr Fermi-LAT Sources Catalog (2FGL; Nolan et al. 2012). As such, most sources identified as $\gamma$-ray-loud in 2LAC are high-confidence statistical associations determined by three association methods using the detected positions and uncertainties of radio/X-ray and $\gamma$-ray emissions within a source's 95\% error ellipse (Ackermann et al. 2011b), along with the physical properties of likely candidates.

The determination of a high-confidence association in 2LAC is also subject to a number of considerations (Ackermann et al. 2011b; Nolan et al. 2012). Candidate gamma-ray sources are "detected" in 2FGL if a region of concentrated flux against its background obtains a test statistic TS $>25$ (corresponding to over $4 \sigma$ significance). There is low availability of spectroscopic information for radio sources in southern declinations. There are in some case multiple associations within a gamma-ray source's $95 \%$ error ellipse. A candidate's gamma-ray spectral intensity may not lie within LAT's energy-dependent sensitivity curve. Candidates with weak radio flux would also have reduced likelihoods of association as they become indistinguishable from other weak radio sources within the LAT error ellipse. These factors would cause bias to higher unassociated AGNs despite possibly having detectable $\gamma$-ray flux. To address these issues, we employ the "clean sample" subset of 2 LAC where sources are excluded if they presented difficulties in the analysis of making an association. We also apply a low threshold to flux densities to account for the possibility of false non-associations below the cutoff.

There is also possible bias introduced by $\gamma$-ray attenuation due to the extragalactic background light (EBL), resulting in a lower rate of association at higher redshifts. Predictions from EBL models show that $\gamma$-rays with energies below $\sim 10 \mathrm{GeV}$ from redshifts up to $z \sim 3$ do not undergo significant attenuation (Stecker et al. 2006; Franceschini et al. 2008; Abdo et al. 2010), and Abdo et al. (2010) find no redshift dependence in the flux ratio of $\gamma$-ray photons $F(>10 \mathrm{GeV}) / F(>1 \mathrm{GeV})$ across blazar subclasses from $z=0$ to above $z=2$. Applying an upper limit to redshift can eliminate the possibility of this bias in our sample.

Furthermore, errors in modeling the diffuse $\gamma$-ray background near the Galactic ridge or in nearby interstellar cloud regions may introduce false associations between $\gamma$-ray and radio emissions belonging to two distinct sources, identifying " $\gamma$-ray-loud" AGNs that are actually $\gamma$-ray-quiet. However, 2LAC selection criteria include only sources with high Galactic latitudes $\left(|b|>10^{\circ}\right)$, eliminating the possibility of false or non-associations occurring near the Galactic ridge.

In review of these factors in $\gamma$-ray detection and source association, our source selection criteria are therefore as follows:

1. identified in RFC;

2. Galactic latitude $|b|>10^{\circ}$;

3. total $4.85 \mathrm{GHz}$ flux density $S_{4.85}>0.8 \mathrm{Jy}$;

4. redshift $z<2$;

5. spectral index $\alpha_{4.85 / \text { low }}>-0.5$;

6. included in clean sample if included in 2LAC;

7. associated with VLBI images in literature.

These criteria define our RFC-based sample, consisting of 351 AGNs in total. The sample contains $151 \gamma$-ray-loud AGNs from the clean sample, a subset of sources from the second LAT catalog that possess high-confidence associations between radio and $\gamma$-ray counterparts (Ackermann et al. 2011b; see Table 1). A $\gamma$-ray-quiet subsample was defined by the 200 AGNs not detected by LAT (see Table 2).

\subsection{The MOJAVE Subsample}

We consider a subsample of the RFC-based sample defined by sources existing in MOJAVE to investigate concerns of sample inhomogeneity (Lister et al. 2009a). The "MOJAVE subsample" is an unbiased subset since the sources included conform to our selection criteria for the RFC-based sample. The sample contains $65 \gamma$-ray-loud AGNs and $29 \gamma$-ray-quiet AGNs, giving a total sample of 94 AGNs. The sources were observed using the Very Long Baseline Array and processed uniformly. The selection criteria used in MOJAVE are also relatively homogeneous, only departing slightly from uniformity in the 
Table 1

Jet-bend Data for $\gamma$-Ray-Loud Radio AGNs

\begin{tabular}{lccccc}
\hline \hline Source & $\begin{array}{c}S_{4.85} \\
(\mathrm{Jy})\end{array}$ & $\begin{array}{c}\phi_{\mathrm{pc}} \\
\left({ }^{\circ}\right)\end{array}$ & $\begin{array}{c}N_{\mathrm{pc}} \\
\left|\theta_{\mathrm{pc}}\right|_{\max }\end{array}$ & References \\
\hline J0049-5738 & 1.338 & $302 /-28$ & 1 & 28 & $10,12^{*}, 14$ \\
$\mathrm{~J} 0051-0650$ & 0.841 & 307 & 0 & 0 & 14 \\
$\mathrm{~J} 0116-1136$ & 1.488 & 325 & 0 & 0 & $2,4,6,14^{*}$ \\
$\mathrm{~J} 0120-2701$ & 1.000 & 156 & 0 & 0 & $6,11,14^{*}, 15$ \\
$\mathrm{~J} 0136+4751$ & 2.016 & 330 & 0 & 0 & $2,4,6,8,9^{*}, 13,14,18$ \\
$\mathrm{~J} 0137-2430$ & 0.956 & 75 & 0 & 0 & $2,6,11^{*}, 14$ \\
$\mathrm{~J} 0141-0928$ & 0.940 & 224 & 0 & 0 & $2,3,6^{*}, 14$ \\
$\mathrm{~J} 0145-2733$ & 0.833 & 53 & 0 & 0 & 14 \\
$\mathrm{~J} 0204-1701$ & 1.350 & 12 & 0 & 0 & $2,4^{*}, 6,14$ \\
$\mathrm{~J} 0204+1514$ & 3.073 & 303 & 0 & 0 & $1,2,4,6,8,9^{*}, 14,18$ \\
\hline
\end{tabular}

Notes. Columns are as follows: (1) J2000 coordinate based name; (2) total 4.85 flux density in Jy, given by the CRATES catalog (Healey et al. 2007); (3) parsec-scale jet position angles, where entries with multiple values separated by slashes denote the bending angles along the jet; (4) number of parsec-scale jet bends; (5) maximum jet-bend angle; (6) VLBI map references (* denotes the reference used to determine jet bends and maximum jet-bending angle where multiple references exist).

References. (1) Bondi et al. 1996; (2) Dodson et al. 2008 and Scott et al. 2004; (3) Fey \& Charlot 1997; (4) Fey \& Charlot 2000; (5) Fey et al. 1996; (6) Fomalont et al. 2000; (7) Henstock et al. 1995; (8) Lee et al. 2008; (9) Lister et al. 2009a (MOJAVE); (10) Ojha et al. 2005; (11) Ojha et al. 2004; (12) Ojha et al. 2010; (13) Pearson \& Readhead 1988; (14) Radio Fundamental Catalog (RFC, rfc_2013d); (15) Shen et al. 1998; (16) Taylor et al. 1994; (17) Taylor et al. 1996; (18) Tingay et al. 1998 (Paper I); (19) Tingay et al. 2002; (20) Xu et al. 1995.

(This table is available in its entirety in a machine-readable form in the online journal. A portion is shown here for guidance regarding its form and content.)

criterion for radio flux density between AGNs at northern $(1.5 \mathrm{Jy})$ and southern $(2.0 \mathrm{Jy})$ declinations to account for differences in instrument sensitivity. The high fidelity imaging of this sample makes it ideal for jet-bend measurements. The sample is also relatively large, which is advantageous from a statistical standpoint. The data from MOJAVE were not available when Paper I was published. All MOJAVE AGNs were imaged at $15 \mathrm{GHz}$ in the period 1994 August to 2007 September (Lister et al. 2009a).

\section{RESULTS AND DISCUSSION}

Von Montigny et al. (1995) suggest two scenarios where jet bending could influence $\gamma$-ray detection in radio-loud AGNs. If $\gamma$-ray emission is produced within an AGN jet, and if the jet bends significantly downstream of the $\gamma$-ray emitting region, there is a possibility that the $\gamma$-ray emission is beamed away from our line of sight (Doppler-dimmed) while radio emission is beamed toward us. In this case we would observe a $\gamma$-rayquiet but radio-loud AGNs. The other possibility is that there may be AGNs with jets either with no bends or with bends upstream of the $\gamma$-ray-emitting region, producing aligned radio and $\gamma$-ray beaming cones. In this case, we would observe a $\gamma$-ray and radio-loud AGNs. Such possibilities may account for $\gamma$-ray observations not otherwise explained by relativistic beaming.

Two other simple scenarios may also be considered. First, objects for which we lie within the $\gamma$-ray beaming cone but not the radio beaming cone. Such sources would be observable to us as $\gamma$-ray-loud, radio-quiet AGNs. However, it is reasonable to assume that substantial radio emission occurs in regions of $\gamma$-ray emission, making this scenario unlikely. Second, AGN jets for which both $\gamma$-ray and radio beaming cones are mis-
Table 2

Jet-bend Data for $\gamma$-Ray-Quiet Radio AGNs

\begin{tabular}{lcrccc}
\hline \hline Source & $\begin{array}{c}S_{4.85} \\
(\mathrm{Jy})\end{array}$ & $\begin{array}{r}\phi_{\mathrm{pc}} \\
\left.{ }^{\circ}\right)\end{array}$ & $N_{\mathrm{pc}}$ & $\begin{array}{c}\left|\theta_{\mathrm{pc}}\right|_{\max } \\
\left(^{\circ}\right)\end{array}$ & References \\
\hline J0006-0623 & 2.463 & 279 & 0 & 0 & $2,3,6,8,9^{*}, 14$ \\
J0010+1724 & 0.989 & 266 & 0 & 0 & $4^{*}, 14$ \\
J0013+4051 & 1.035 & 331 & 0 & 0 & $2,3,6,14^{*}, 20$ \\
J0019+7327 & 1.583 & 144 & 0 & 0 & $2,3,6,8,9^{*}, 13,14,18$ \\
J0024-4202 & 2.036 & 297 & 0 & 0 & 14 \\
J0029+3456 & 1.182 & 56 & 0 & 0 & $3,6,14^{*}, 16$ \\
J0038+4137 & 1.144 & 113 & 0 & 0 & $6^{*}, 7,14$ \\
J0042+2320 & 1.604 & 118 & 0 & 0 & $2,4,6,14^{*}$ \\
J0051-4226 & 0.926 & 40 & 0 & 0 & $6^{*}, 14$ \\
J0057+3021 & 0.914 & 309 & 0 & 0 & \\
\hline
\end{tabular}

Notes. Columns are as follows: (1) J2000 coordinate based name; (2) total 4.85 flux density in Jy, given by the CRATES catalog (Healey et al. 2007); (3) parsec-scale jet position angles, where entries with multiple values separated by slashes denote the bending angles along the jet; (4) number of parsec-scale jet bends; (5) maximum jet-bend angle; (6) VLBI map references ( $^{*}$ denotes the reference used to determine jet bends and maximum jet-bending angle where multiple references exist).

References. (1) Bondi et al. 1996; (2) Dodson et al. 2008 and Scott et al. 2004; (3) Fey \& Charlot 1997; (4) Fey \& Charlot 2000; (5) Fey et al. 1996; (6) Fomalont et al. 2000; (7) Henstock et al. 1995; (8) Lee et al. 2008; (9) Lister et al. 2009a; (10) Ojha et al. 2005; (11) Ojha et al. 2004; (12) Ojha et al. 2010; (13) Pearson \& Readhead 1988; (14) Radio Fundamental Catalog (RFC, rfc_2013d); (15) Shen et al. 1998; (16) Taylor et al. 1994; (17) Taylor et al. 1996; (18) Tingay et al. 1998 (Paper I); (19) Tingay et al. 2002; (20) Xu et al. 1995.

(This table is available in its entirety in a machine-readable form in the online journal. A portion is shown here for guidance regarding its form and content.)

aligned with our line of sight. These sources will likely not be identified in $\gamma$-rays and may appear as weak radio sources.

In our approach, the number of parsec-scale jet bends and the maximum parsec-scale jet-bending angles are recorded for AGNs with VLBI images that present a discernible jet structure from which jet-bend data may be extracted. The hypothesis of von Montigny et al. (1995) allows the possibility that jet bending could be significant on kiloparsec scales. However, neither Bower et al. (1997) nor Paper I find a significant difference in the parsec-to-kiloparsec misalignment angle between $\gamma$-rayloud and $\gamma$-ray-quiet populations. In addition, Paper I does not find a statistically significant difference in the number of kiloparsec-scale jet bends. More recent investigations assert that $\gamma$-ray emission originates in the parsec-scale region, near the core, although the precise region continues to be discussed (Jorstad et al. 2001; Lähteenmäki \& Valtaoja 2003; Sikora et al. 2009). In the scenario proposed in these studies, both $\gamma$-ray and radio emission may be produced far upstream of any kiloparsecscale bending, allowing the detection of both kinds of emission regardless of the existence of kiloparsec bending. Kiloparsecscale bending does not appear to impact $\gamma$-ray detection in AGNs, consistent across a number of studies, and is not explored here.

We applied the following method to our jet-bending measurements. For a given AGN, we compared VLBI images available from the RFC and in other major surveys. Considering the resolution and common features between epochs, we determined the AGN's jet structure that could be discernable by eye. In this process, we assume that an abrupt change in the apparent direction of a jet, associated with a "shock" region of high flux density, corresponds to the bending between two jet sections. We selected the best VLBI image to derive its jet properties, 

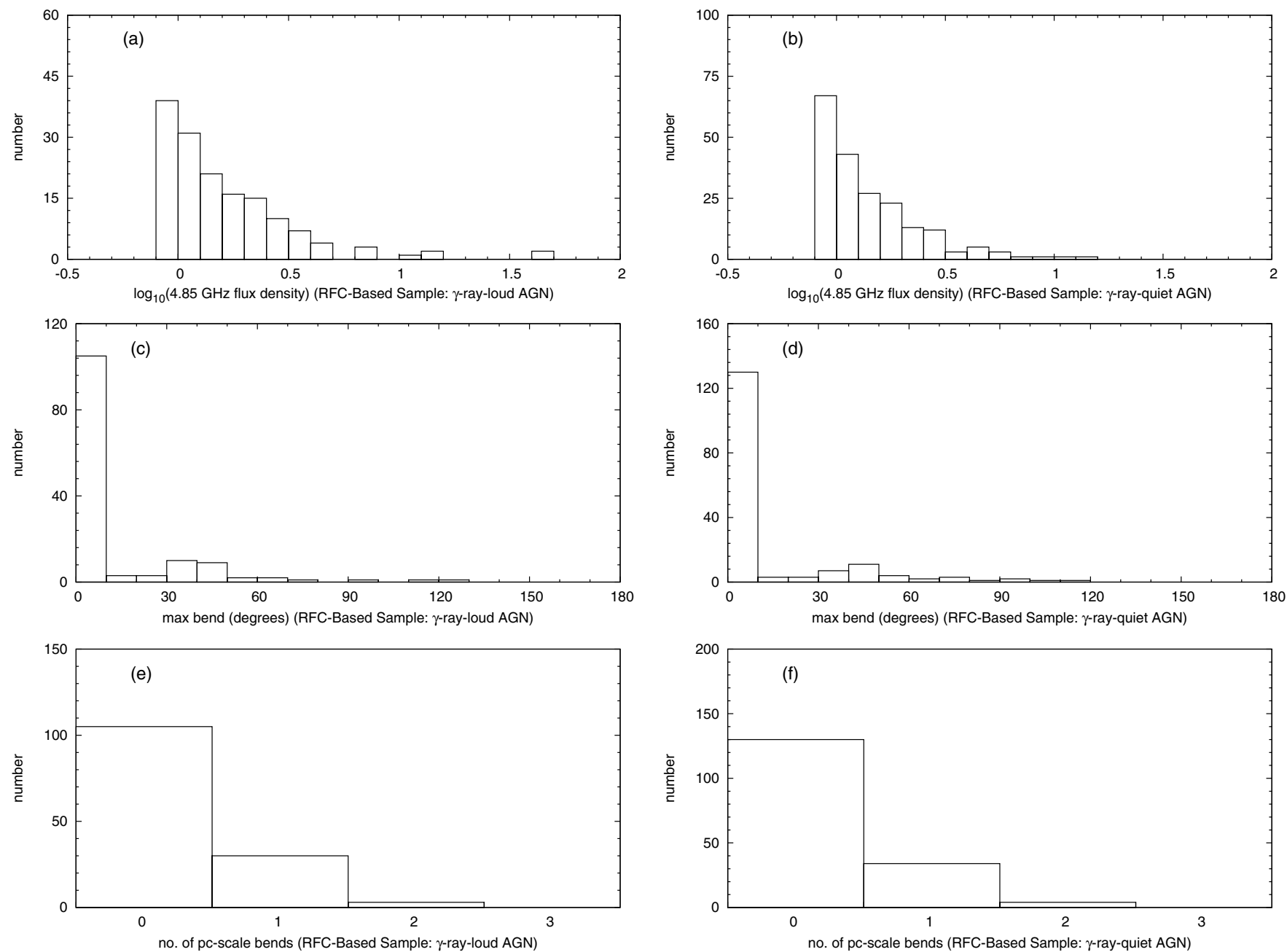

Figure 1. Distributions of (a)-(b) $\log _{10}(4.85 \mathrm{GHz}$ flux density), (c)-(d) maximum bend and, (e)-(f) number of bends for $\gamma$-ray loud and $\gamma$-ray-quiet radio AGNs of the RFC-based sample.

with preference always given to available MOJAVE images due to their superior image quality. The initial angle of the jet from the core was measured east of north in the equatorial coordinate system. The angles of any existing bends in the jet are then measured (in the same sense) with respect to the previous jet trajectory closer to the core. The number of bends and the maximum jet-bending angle were then recorded for each source, shown in Tables 1 and 2.

There are a number of factors affecting an AGN's derived jet properties. Inhomogeneity is introduced from the variable resolution and sensitivity of VLBI instrumentation, the choice of radio frequency for imaging, and different image epochs for different sets of images associated with a particular AGN. As such, the low fidelity of some VLBI images may affect the consistency of jet measurements across the whole sample. Despite this, using the best available VLBI images for each AGN will provide the most accurate jet-bending properties to retest the jet-bending hypothesis. The ability to resolve jet components also diminishes with decreasing radio flux density. In weak radio sources, this is particularly evident in the reduced ability to resolve low flux density jet components (and therefore jet bending) at large distances from the core. Deflections in jets may not be obvious at small jet-bending angles, and where there are jet bends, they may not occur at a single, welldefined point along the jet. The comparison between multiple VLBI images of AGNs across different imaging frequencies and epochs also allows us to obtain jet properties that reflect the jet structure from the observer's point of view as accurately as possible. Furthermore, bright individual features with different trajectories within or around a jet may also give the false appearance of jet bending in jet structure in certain imaging epochs (Homan 2012). Comparing VLBI images at different epochs assists in identifying bright regions that may temporarily distort the appearance of the jet structure. Measurements are subject to image fidelity and morphological interpretation; however, these considerations should provide a robust result when testing the significance of jet bending in $\gamma$-ray detection compared to the findings of previous, more-limited studies.

We employ a number of statistical tests to explore differences between $\gamma$-ray-loud and $\gamma$-ray-quiet populations in our sample. A two-sided Kolmogorov-Smirnov (K-S) two-sample test was used to determine the statistical significance of differences at $4.85 \mathrm{GHz}$ flux density and maximum jet-bend angle distributions between $\gamma$-ray-loud and $\gamma$-ray-quiet AGN populations. Since the values for the number of jet bends are discrete, a $\chi^{2}$ test was applied to characterize differences between the distributions in this case. Thirteen $\gamma$-ray-loud and $32 \gamma$-ray-quiet sources did not show discernible jets and thus were omitted from the statistical testing of jet properties. For all tests, the difference between sample populations is considered significant for a given property when, assuming the null hypothesis represents both samples being drawn from the same parent population, the 

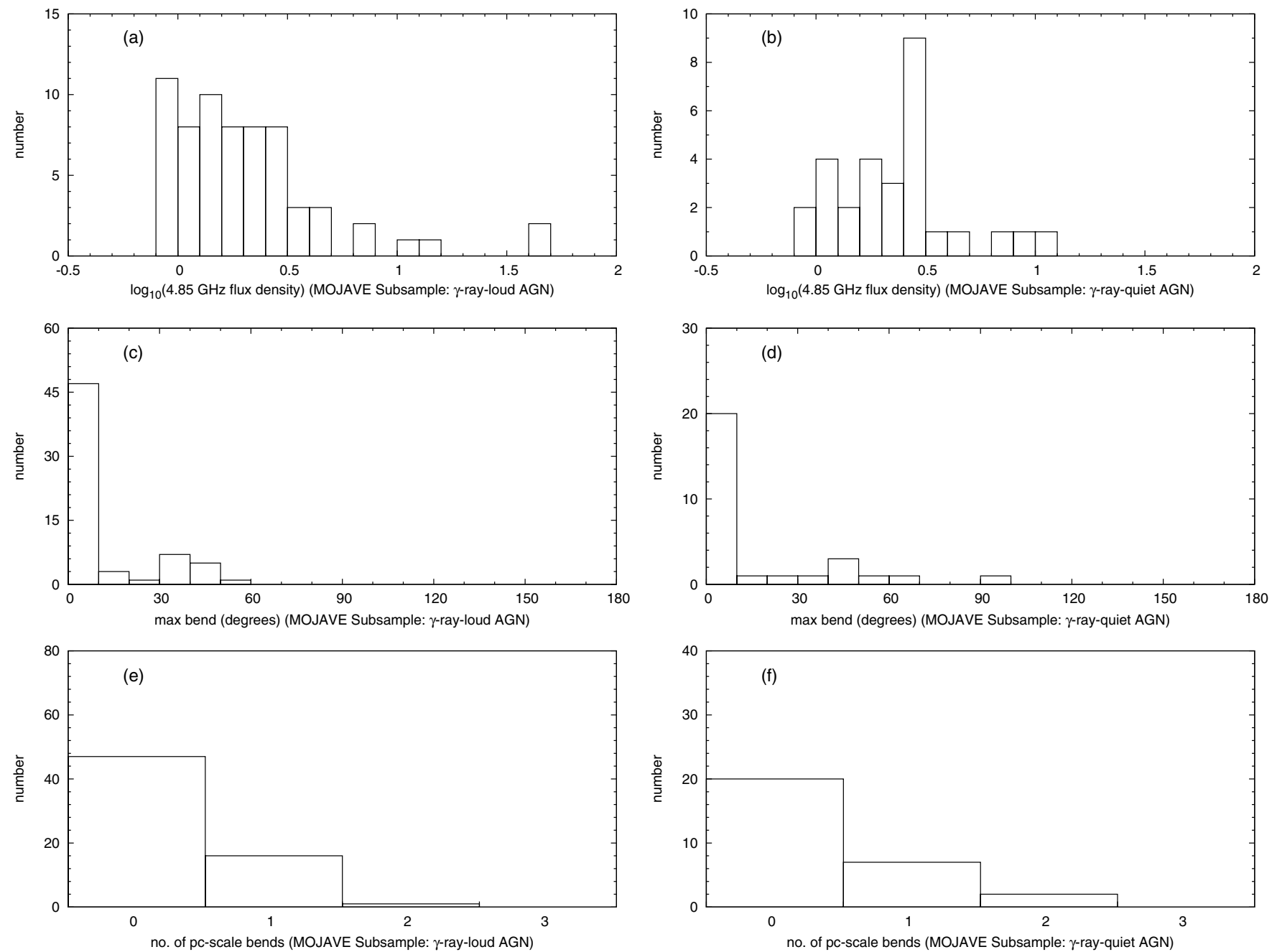

Figure 2. Distributions of (a)-(b) $\log _{10}(4.85 \mathrm{GHz}$ flux density), (c)-(d) maximum bend, and (e)-(f) number of bends for $\gamma$-ray loud and $\gamma$-ray-quiet radio AGNs of the MOJAVE subsample.

Table 3

Statistical Results (K-S and $\chi^{2}$ Tests)

\begin{tabular}{lccc}
\hline \hline Source Property & Statistical Test & $p\left(H_{0}\right)$ & Significant \\
\hline RFC-based sample & & & \\
$\quad$ Total 4.85 GHz flux density & K-S & 0.121 & No \\
Maximum pc-scale jet bend & K-S & 0.998 & No \\
Number of pc-scale jet bends & $\chi^{2}$ & 0.945 & No \\
\hline Lister et al. 2009a (MOJAVE) sample & & & \\
$\quad$ Total 4.85 GHz flux density & K-S & 0.145 & No \\
Maximum pc-scale jet bend & K-S & 0.948 & No \\
Number of pc-scale jet bends & $\chi^{2}$ & 0.402 & No \\
\hline
\end{tabular}

Note. A result is considered significant when, assuming the null hypothesis holds, the probability of observing a test statistic at least as extreme as the one obtained $p\left(H_{0}\right)$ is less than 0.05 ( $95 \%$ confidence level).

probability of observing a test statistic at least as extreme as the one obtained $\left(p\left(H_{0}\right)\right)$ is less than $0.05(95 \%$ confidence that the null hypothesis can be rejected). These results are summarized in Table 3.

\subsection{The RFC-based Sample}

Figures 1(a) and (b) show similar distributions for AGN total flux densities at $4.85 \mathrm{GHz}$. A K-S test determines that the probability that the two samples have been drawn from the same parent population is $p\left(H_{0}\right)=0.121$, finding no statistically significant difference between the two distributions. The result gives confidence that there are no biases in LAT detections that may skew jet-bending statistics.

We now consider the jet-bending statistics of $\gamma$-ray-loud and $\gamma$-ray-quiet samples. The distributions between samples for the number of jet bends (Figures 1(e) and (f)) show no significant differences. A $\chi^{2}$ test finds a statistically insignificant probability of $p\left(H_{0}\right)=0.945\left(\chi^{2}=0.112\right.$ with two degrees of freedom). Similarly, histograms for the parsec-scale maximum jet-bend angle (Figures 1(c) and (d)) demonstrate no significant difference between the two populations. The probability that the two samples belong to the same parent population is $p\left(H_{0}\right)=0.998$.

\subsection{The MOJAVE Subsample}

We find that there are no statistically significant differences between $\gamma$-ray-loud and $\gamma$-ray-quiet AGN populations for any of the tested properties. The results of the statistical tests on the MOJAVE subsample are given in Table 3. The $\gamma$-ray-loud and $\gamma$-ray-quiet distributions for these properties are shown in Figure 2. 


\section{SUMMARY}

We have investigated the suggestion that $\gamma$-ray-quiet AGNs have a greater tendency for jet bending than $\gamma$-ray-loud AGNs (von Montigny et al. 1995). We present a statistical analysis of a large sample of AGNs that represents the population of radio-loud AGNs, using data from the Fermi LAT instrument. We also conducted an analysis of a homogeneous subsample (MOJAVE) to address the inhomogeneity of selection criteria and VLBI image quality in the RFC-based sample.

Our analysis of the RFC-based sample shows that jet bending does not play a significant role in predicting $\gamma$-ray detectability in radio-loud AGNs. As such our results do not support the suggestion of von Montigny et al. (1995). This conclusion is in contradiction to Paper I, and supports the findings of Linford et al. (2012). The contrast of results obtained by new data with those of Paper I reveals the strong dependence on the fidelity and homogeneity of VLBI images in jet-bend measurements. The limited quality and homogeneity of VLBI images available at the time of study appears to be the reason for the reported conclusions in Paper I.

The results of the MOJAVE subsample generally support the conclusions drawn from the RFC-based sample. Statistically insignificant results were found for both the number of parsecscale jet bends and maximum parsec-scale jet bend, indicating that the RFC-based sample is not adversely affected by VLBI image inhomogeneity and giving additional confidence to the null result.

We thank the referee for providing helpful comments and suggestions. This research has made use of data from the Radio Fundamental Catalog (RFC) maintained by L. Petrov, solution rfc_2013d (unpublished, available online at http://astrogeo.org/vlbi/solutions/rfc_2013d); the MOJAVE database, maintained by the MOJAVE team (Lister et al. 2009a); the CRATES catalog (Healey et al. 2007); and the NASA/IPAC Extragalactic Database (NED), operated by the Jet Propulsion Laboratory, California Institute of Technology, under contract with the National Aeronautics and Space Administration.

\section{REFERENCES}

Abdo, A. A., Ackermann, M., Ajello, M., et al. 2010, ApJ, 723, 1082 Ackermann, M., Ajello, M., Allafort, A., et al. 2011a, ApJ, 741, 30 Ackermann, M., Ajello, M., Allafort, A., et al. 2011b, ApJ, 743, 171 Aller, M. F., Aller, H. D., \& Hughes, P. A. 1996, in IAU Symp. 175, Extragallactic Radio Sources, ed. R. D. Ekers, C. Fanti, \& L. Padrielli (Dordrecht: Kluwer), 283

Balsara, D. S., \& Norman, M. L. 1992, ApJ, 393, 631

Beasley, A. J., Gordon, D., Peck, A. B., et al. 2002, ApJS, 141, 13 (RFC)

Bondi, M., Padrielli, L., Fanti, R., et al. 1996, A\&A, 308, 415

Bower, G. C., Backer, D. C., Wright, M., et al. 1997, ApJ, 484, 118

Dodson, R., Fomalont, E. B., Wiik, K., et al. 2008, ApJS, 175, 314

Fey, A. L., \& Charlot, P. 1997, ApJS, 111, 95

Fey, A. L., \& Charlot, P. 2000, ApJS, 128, 17

Fey, A. L., Clegg, A. W., \& Fomalont, E. B. 1996, ApJS, 105, 299

Fichtel, C. E., Bertsch, D. L., Chiang, J., et al. 1994, ApJS, 94, 551

Fomalont, E. B., Frey, S., Paragi, Z., et al. 2000, ApJS, 131, 95

Fomalont, E. B., Petrov, L., MacMillan, D. S., Gordon, D., \& Ma, C. 2003, AJ, 126, 2562 (RFC)

Franceschini, A., Rodighiero, G., \& Vaccari, M. 2008, A\&A, 487, 837

Hartman, R. C., Bertsch, D. L., Bloom, S. D., et al. 1999, ApJS, 123, 79
Healey, S. E., Romani, R. W., Taylor, G. B., et al. 2007, ApJS, 171, 61 (CRATES) Henstock, D. R., Browne, W. A., Wilkinson, P. N., et al. 1995, ApJS, 100, 1

Homan, D. C. 2012, IJMPS, 8, 163

Hughes, P. A., Aller, M. F., \& Aller, H. D. 2011, ApJ, 735, 81

Immer, K., Brunthaler, A., Reid, M. J., et al. 2011, ApJS, 194, 25 (RFC)

Impey, C. 1996, AJ, 112, 2667

Jorstad, S. G., Marscher, A. P., Mattox, J. R., et al. 2001, ApJ, 556, 738

Kellermann, K. I., Lister, M. L., Homan, D. C., et al. 2004, ApJ, 609, 539

Kovalev, Y. Y., Aller, H. D., Aller, M. F., et al. 2009, ApJL, 696, L17

Kovalev, Y. Y., Petrov, L., Fomalont, E. B., \& Gordon, D. 2007, AJ, 133, 1236 (RFC)

Lähteenmäki, A., \& Valtaoja, E. 1999, in ASP Conf. Ser. 159, BL Lac Phenomenon, ed. L. O. Takalo \& A. Sillanpää (San Francisco, CA: ASP), 213

Lähteenmäki, A., \& Valtaoja, E. 2003, ApJ, 590, 95

Lebedev, S. V., Ampleford, D., Ciardi, A., et al. 2004, ApJ, 616, 988

Lee, S.-S., Lobanov, A. P., Krichbaum, T. P., et al. 2008, AJ, 136, 159

León-Tavares, J., Valtaoja, E., Tornikoski, M., Laḧteenmäki, A., \& Nieppola, E. 2011, A\&A, 532, A146

Linford, J. D., Taylor, G. B., Romani, R. W., et al. 2011, ApJ, 726, 16

Linford, J. D., Taylor, G. B., Romani, R. W., et al. 2012, ApJ, 744, 177

Lister, M. L., Aller, H. D., Aller, M. F., et al. 2009a, AJ, 137, 3718 (MOJAVE)

Lister, M. L., Aller, M. F., Aller, H., et al. 2011, ApJ, 742, 27

Lister, M. L., Cohen, M. H., Homan, D. C., et al. 2009b, AJ, 138, 1874

Mattox, J. R., Schachter, J., Molnar, L., Hartman, R. C., \& Patnaik, A. R. 1997a, ApJ, 481, 95

Mattox, J. R., Wagner, S. J., Malkan, M., et al. 1997b, ApJ, 476, 692

Mücke, A., Pohl, M., Reich, P., et al. 1997, A\&A, 320, 33

Nieppola, E., Tornikoski, M., Valtaoja, E., et al. 2011, A\&A, 535, A69

Nolan, P. L., Abdo, A. A., Ackermann, M., et al. 2012, ApJS, 199, 31

Ojha, R., Fey, A. L., Charlot, P., et al. 2005, AJ, 130, 2529

Ojha, R., Fey, A. L., Johnston, K. J., et al. 2004, AJ, 127, 3609

Ojha, R., Kadler, M., Böck, M., et al. 2010, A\&A, 519, A45

Pearson, T. J., \& Readhead, A. C. S. 1988, ApJ, 328, 114

Petrov, L. 2011, AJ, 142, 105 (RFC)

Petrov, L. 2012, MNRAS, 416, 1097 (RFC)

Petrov, L. 2013, AJ, 146, 5 (RFC)

Petrov, L., Kovalev, Y. Y., Fomalont, E., \& Gordon, D. 2005, AJ, 129, 1163 (RFC)

Petrov, L., Kovalev, Y. Y., Fomalont, E. B., \& Gordon, D. 2006, AJ, 131, 1872 (RFC)

Petrov, L., Kovalev, Y. Y., Fomalont, E. B., \& Gordon, D. 2008, AJ, 136, 580 (RFC)

Petrov, L., Kovalev, Y. Y., Fomalont, E. B., \& Gordon, D. 2011a, AJ, 142, 35 (RFC)

Petrov, L., Phillips, C., Bertarini, A., Murphy, T., \& Sadler, E. M. 2011b, MNRAS, 414, 2528 (RFC)

Petrov, L., \& Taylor, G. B. 2011, AJ, 142, 89 (RFC)

Pushkarev, A. B., Kovalev, Y. Y., \& Lister, M. L. 2010, ApJL, 722, L7

Pushkarev, A. B., Kovalev, Y. Y., Lister, M. L., \& Savolainen, T. 2009, A\&A, 507, L33

Richards, J. L., Max-Moerbeck, W., Pavlidou, V., et al. 2011, ApJS, 194, 29

Salamon, M. H., \& Stecker, F. W. 1994, ApJL, 430, L21

Savolainen, T., Homan, D. C., Hovatta, T., et al. 2010, A\&A, 512, A24

Scott, W. K., Fomalont, E. B., Horiuchi, S., et al. 2004, ApJS, 155, 33

Shen, Z.-Q., Wan, T.-S., Moran, J. M., et al. 1998, AJ, 115, 1357

Sikora, M., Stawarz, Ł., Nalewajko, K., \& Madejski, G. M. 2009, ApJ, 704, 38

Stecker, F. W., Malkan, M. A., \& Scully, S. T. 2006, ApJ, 648, 774 (erratum, 658, 1392 [2007])

Taylor, G. B., Healey, S. E., Helmboldt, J. F., et al. 2007, ApJ, 671, 1355

Taylor, G. B., Vermeulen, R. C., Pearson, T. J., et al. 1994, ApJS, 95, 345

Taylor, G. B., Vermeulen, R. C., Readhead, A. C. S., et al. 1996, ApJS, 107,37

Tingay, S. J., Jauncey, D. L., King, E. A., et al. 2003, PASJ, 55, 351

Tingay, S. J., Jauncey, D. L., Reynolds, J. E., et al. 1996, AJ, 111, 718

Tingay, S. J., Murphy, D. W., \& Edwards, P. G. 1998, ApJ, 500, 673 (Paper I)

Tingay, S. J., Reynolds, J. E., Tzioumis, A. K., et al. 2002, ApJS, 141, 311

von Montigny, C., Bertsch, D. L., Chiang, J., et al. 1995, A\&A, 299, 680

Xu, W., Readhead, A. C. S., Pearson, T. J., Polatidis, A. G., \& Wilkinson, P. N. 1995, ApJS, 99, 297 